Hadza hunter-gatherers disagree on perceptions of moral character

\author{
Kristopher M. Smith
}

Coren L. Apicella

Department of Psychology, University of Pennsylvania, 3720 Walnut Street, Philadelphia, PA 19104

Corresponding author: Coren L. Apicella, capicella@psych.upenn.edu

Word count: 4999

Kristopher Smith is a doctoral candidate in the Department of Psychology at the University of Pennsylvania. He studies the origins of cooperation and morality, by conducting field research among the Hadza of Tanzania to answer these questions.

Coren Apicella is an Assistant professor of Psychology at the University of Pennsylvania. She studies how biology and culture work jointly, and separately, to shape individual and social decisions, from mate choice, to cooperation, to competition.

Acknowledgements: The project was supported by the Templeton Religion Trust as a sub-grant through the Moral Beacons Project. We thank Endeko Endeko, Deus Haraja, and Ibrahim Mabulla for their assistance in data collection, and Audax Mabulla for his administrative support. We also thank Joyce Zhao for recommending JAGS and Corey Cusimano for helpful feedback on the manuscript. Finally, we thank the Hadza participants. 


\begin{abstract}
To the extent that moral character is grounded in stable and observable truths, there should exist agreement between people in their judgements of others' character. In Western populations, this agreement is found. We examine whether this is universal in Hadza hunter-gatherers of Tanzania. Ninety-four judges ranked their campmates on global character and relevant character traits for a total of 824 observations. Judges disagreed on rankings of global character, generosity, and honesty, but agreed more on hard work and hunting ability. Individual rankings on specific traits predicted character evaluations. There was agreement between judges on the extent to which generosity and hard work related to character. These findings suggest that Hadza have shared beliefs about what traits constitute character, but disagree on which of their campmates exhibit these traits. We discuss these findings in light of other research suggesting that stable moral dispositions may not be universal.

Keywords: Hadza, hunter-gatherers, cross-cultural, moral character, person perception, reputation
\end{abstract}




\section{Hadza hunter-gatherers disagree on perceptions of moral character}

In Western societies, evaluation of moral character is an important component of person perception (Goodwin, 2015; Goodwin, Piazza, \& Rozin, 2014). When learning about a new person, Italian undergraduates seek information about whether they are trustworthy (Brambilla, Rusconi, Sacchi, \& Cherubini, 2011). When identifying features and traits most relevant to identity, US online workers consider morality to be an essential component (Strohminger \& Nichols, 2014). And when US undergraduates consider what attributes a partner should have in different types of relationships, morally relevant features, such as trustworthiness, are most important (Cottrell, Neuberg, \& Li, 2007). This makes sense because a person's character is used to infer their intentions and whether they would help or hinder one's goals (Landy, Piazza, \& Goodwin, 2016). Indeed, people use information about moral character to decide who to interact and cooperate with (Everett, Faber, Savulescu, \& Crockett, 2018; Martin \& Cushman, 2015; van der Lee, Ellemers, Scheepers, \& Rutjens, 2017).

Despite the importance of moral character in person perception, some have argued that people in Western populations do not have stable moral dispositions (Doris, 2002; Harman, 2003). Social psychologists and philosophers have used classic findings from social psychology, such as the bystander effect (Darley \& Latané, 1968; Latané \& Darley, 1968) or the good Samaritan experiment (Darley \& Batson, 1973), to argue against the existence of moral character and that moral behavior is determined wholly by the situation (Doris, 2002; Harman, 2003). One way to determine whether people behave similarly across situations is to examine agreement between independent observers. Because different observers are likely to interact with the target in different situations, if they agree in their evaluations, it then suggests there is a stable disposition that is being observed (Kenrick \& Funder, 1988). So, if independent observers have 
similar perceptions of targets' moral character, then it provides evidence for the existence of moral character.

Western participants generally agree on perceptions of moral character. In a US community sample, self-report and informant ratings of morally-relevant traits, such as honesty or guilt-proneness, moderately correlate (Cohen, Panter, Turan, Morse, \& Kim, 2013). Independent observers in US community samples also agree on global evaluations of moral character, as well as specific moral traits and trait profiles (Helzer et al., 2014). And US online workers agree on moral traits displayed by cultural figures, even across the US political divide (Frimer, Biesanz, Walker, \& MacKinlay, 2013). Again, this agreement is used as evidence that moral character exists.

Samples in studies of moral psychology have largely been drawn from Western, Educated, Industrialized, Rich, and Democratic_-or WEIRD—societies (Ellemers, van der Toorn, Paunov, \& van Leeuwen, 2019; Henrich, Heine, \& Norenzayan, 2010). Despite this, the importance of moral character in identity and person perception is theorized to be universal (Strohminger, Knobe, \& Newman, 2017). Most often, when moral psychology is examined in other cultures, the emphasis is on the content of moral norms and the shared or unique prescriptions and prohibitions across cultures (Curry, Mullins, \& Whitehouse, 2019; Haidt, Koller, \& Dias, 1993; Purzycki et al., 2018). Yet, when research has looked at processes in moral judgments, important differences have been found. For example, whether a wrong is done intentionally is an important distinction in moral judgments among Western populations, presumably because it reveals information about moral character (Landy \& Uhlmann, 2018). However, unintentional violations are judged as wrong as intentional violations in some cultures, including the Hadza and South Pacific islanders (Barrett et al., 2016; McNamara, Willard, 
Norenzayan, \& Henrich, 2019). To our knowledge, no research has been conducted on perceptions of moral character in small-scale societies.

There are reasons to suspect important differences in moral character and its perception in small-scale societies. First, there is some evidence for less personality variation in nonWEIRD societies. For example, personality traits in the Tsimané forager-horticulturalists of Bolivia do not cluster into five distinct factors, but rather two, and there is less variation within those factors compared to Western samples (Gurven, von Rueden, Massenkoff, Kaplan, \& Vie, 2013). And in fact, across 55 nations, populations with fewer economic opportunities to specialize have less variation in personality traits (Lukaszewski, Gurven, von Rueden, \& Schmitt, 2017). To the extent that there is a relationship between personality traits and moral character (Thalmayer, Saucier, Srivastava, Flournoy, \& Costello, 2019), we might then similarly expect less variation in morally-relevant character traits. Second, there is no evidence for stable variation in generous dispositions in small-scale societies. In longitudinal data among the Hadza, contributions to a public good game were not predicted by previous contributions, but rather the contributions of an individual's campmates (Smith, Larroucau, Mabulla, \& Apicella, 2018). Here, local norms governing generosity may reduce individual variation in moral behavior, leading to a lack of agreement on perceptions of moral character.

In the current study, we examine perceptions of moral character among the Hadza of Tanzania, one of the last remaining hunter-gatherer groups. The Hadza are an ideal population because they live in small groups of known individuals where behavior is observable, and because of their harsh environment, knowing who is moral would be important. We examine agreement on these perceptions in two ways. First, do Hadza agree on who has moral character? And second, do Hadza agree on what traits contribute to global moral character? To answer these 
questions, we ask the Hadza to rank their campmates on moral character, as well as specific traits of hard work, generosity, and honesty. We examine the consensus within each camp on rank orderings for each trait to answer the first question. We examine the relationship between the specific traits and global character rankings and the variation between Hadza on the importance of the specific traits in determining global character to answer the second question.

\section{Method}

\section{Population}

The Hadza are nomadic hunter-gatherers living in rural north Tanzania around Lake Eyasi. There are about 300 Hadza who still practice a foraging lifestyle (Marlowe, 2010). The Hadza have a sexual division of labor; men hunt for game, such as giraffe, impala, or zebra, and collect honey, while women gather plant items, such as tubers and berries. Food brought back to the camp that requires extended processing, such as meat and tubers, are distributed across the entire camp. Food and fresh water is scarce; about $80 \%$ of Hadza report concerns about whether there will be enough food in the next month (Apicella, 2018). Other threats to Hadza include high rates of disease and infection, and less commonly, attacks from predators, such as lions, hyenas, leopards, and snakes. Approximately $40 \%$ of children born will not live to reach the age of five (Blurton-Jones, 2016).

The Hadza live in temporary camps of about 30 adults and children, usually consisting of two to three unrelated nuclear families. The Hadza a have multilocal residence pattern — men and women are equally likely to live with kin, though the average genetic relatedness for both sexes within camps is low (Blurton-Jones, 2016; Hill et al., 2011). Camps move location every 4 to 6 weeks as local resources are depleted, and people frequently join new camps as they please. Repeat interaction rates are low in the Hadza (Hill, Wood, Baggio, Hurtado, \& Boyd, 2014), and 
from year-to-year, individuals are only living with about $20 \%$ of their previous campmates (Smith et al., 2018).

Though the Hadza have strong norms of food sharing, they do try to cheat these norms when possible. In economic games that measure rule-bending, Hadza will cheat to benefit themselves at the expense of their campmates (Apicella, 2018). Anecdotally, Hadza will sometimes try to bring in meat under the cover of darkness to avoid sharing with others and will sometimes even ask to hide carcasses under a researcher's vehicle (Marlowe, 2010). Hadza historically have little experience with centralized institutions involving punishment (e.g., police and courts). In economic games, the Hadza have low-rates of second- and third-party punishment (Henrich, 2006). And though most Hadza report believing in god (Haine or sometimes Ishoko), few attribute to Haine moralistic concerns or the power to detect and punish norm violations (Apicella, 2018). Thus, there are opportunities for the Hadza to show variation in moral behavior and for others to witness it, though observed deviations may not be punished.

\section{Sample}

The first author and research assistants visited 12 camps $^{1}$ during the dry-season in August-September 2016 using a snowball sampling procedure; after visiting one camp, members of that camp would direct us toward the nearest camp. We collected data until we could not identify any more camps. The number of adults in each camp ranged from three to twelve. We interviewed 94 judges who ranked 95 subjects (one participant left camp after his photo was taken but before he was interviewed) for a total of $N=824$ observations. Two judges $(n=20$ observations) were removed because they refused to rank their campmates on honesty, stating

\footnotetext{
${ }^{1}$ We collected data from a thirteenth camp that had 36 adults; judges found the task of ranking this many people arduous. We had judges in this camp rank the top twelve campmates on each trait instead. However, the analyses used here require people to be ranking the same set of participants, so we did not use these data. Data for this camp were never entered or analyzed.
} 
that everyone in their camp is a liar. Another two observations were removed because of an error in recording the data. The final sample included 95 subjects $(n=46$ men, mean age $M=39.8$ years-old, $n=68$ married) ranked by 92 judges for $n=802$ observations.

\section{Procedure}

We asked judges to rank their campmates on generosity ("Who shares the most food?"), hard work or effort ("Who works the hardest to get food?"), honesty ("Who tells the fewest lies?"), and global moral character ("Who has the best heart?"). We asked about generosity, hard work, and honesty because in previous research when Hadza are asked to free list traits that make a good person these were some of the nominated traits (Purzycki et al., 2018). We used "good heart" as a global character judgment because this was the most common response in the free list task and the Hadza seem to equate good heart with being a good person. We also asked them to rank men on who is the best hunter ("Who is the best hunter?"), and which we include as a non-moral comparison. Finally, we asked them who they prefer as campmates, but do not include that data here.

To have judges rank their campmates, we took headshot photographs of all the consenting adults in a camp using a Fujifilm Instax Mini 90 Classic Instant Film Camera which printed $1.8 \times 2.4$ inch images. We then conducted private interviews in Swahili with each adult. A research assistant would shuffle the photographs and lay them in front of the judge. The assistant would then ask the judge, "Who shares the most food?" After the judge picked a photograph, the assistant would remove the photograph, shuffle the remaining photographs, and repeat the question. This was repeated until all campmates were ranked on that trait. The entire process was then repeated for each trait. The Hadza have previously done this task multiple times 
to rank campmates on hunting ability (Apicella, 2014; Smith, Olkhov, Puts, \& Apicella, 2017; Stibbard-Hawkes, Attenborough, \& Marlowe, 2018).

\section{Analysis}

We used Bayesian regression models for inferential analyses. Bayesian analyses compute the probability the observed data are generated by a hypothesized parameter value, conditional on the model assumptions and prior probabilities (Kruschke \& Liddell, 2018a; McElreath, 2016). This produces a distribution of probability values across a range of possible parameter values. This allows us to describe the estimated strength of the relationship and the uncertainty around the estimate, rather than focus on null-point hypothesis testing (Kruschke \& Liddell, 2018b). In our analyses we use weakly regularizing priors; these are conservative priors that are skeptical of large associations and that restrict the estimates to more plausible values (McElreath, 2016).

We analyzed the data in R (R, 2017) using the 'rjags' (Plummer, 2018) and 'brms' (Bürkner, 2017) packages. Both packages use Monte Carlo Markov Chains to draw samples from the posterior distributions, drawing more samples from regions with higher probabilities to estimate the posterior distribution; the packages use different algorithms to explore the distribution. We also used the 'tidyverse' (Wickham, 2017), 'tidybayes' (Kay, 2018), 'ggridges' (Wilke, 2018), and 'irr' (Gamer, Lemon, \& Fellows, 2012) packages.

\section{Ethics and transparency}

Research protocols were approved by the Institutional Review Board at the University of Pennsylvania and the Tanzania Commission for Science and Technology. Analyses, and predictions were not pre-registered. De-identified data, scripts, and output are available at https://osf.io/4jms9/. 


\section{Results}

\section{Do Hadza agree on who is moral?}

We examined the extent to which Hadza agree on the ranking of each trait by computing a Kendall's $W$ for each trait for each camp. Kendall's $W$ is a coefficient of consensus for ranking data, ranging from zero to one, with zero indicating no agreement between raters (Zar, 1996). A suggested benchmark for Kendall's $W$ is that 0.5 indicates moderate agreement and 0.3 indicates weak agreement. The observed values are presented in Figure 1. The median value for good heart was 0.19 and ranged from 0.04 to 0.31 , the median value for effort was 0.25 and ranged from 0.07 to 0.60 , the median value for generosity was 0.19 and ranged from 0.00 to 0.48 , the median value for honesty was 0.19 and ranged from 0.10 to 0.44 , and the median value for effort was 0.40 and ranged from 0.16 to 0.63 . The median values for effort, generosity, honesty, and good heart all fall below the suggested benchmark for weak agreement. 


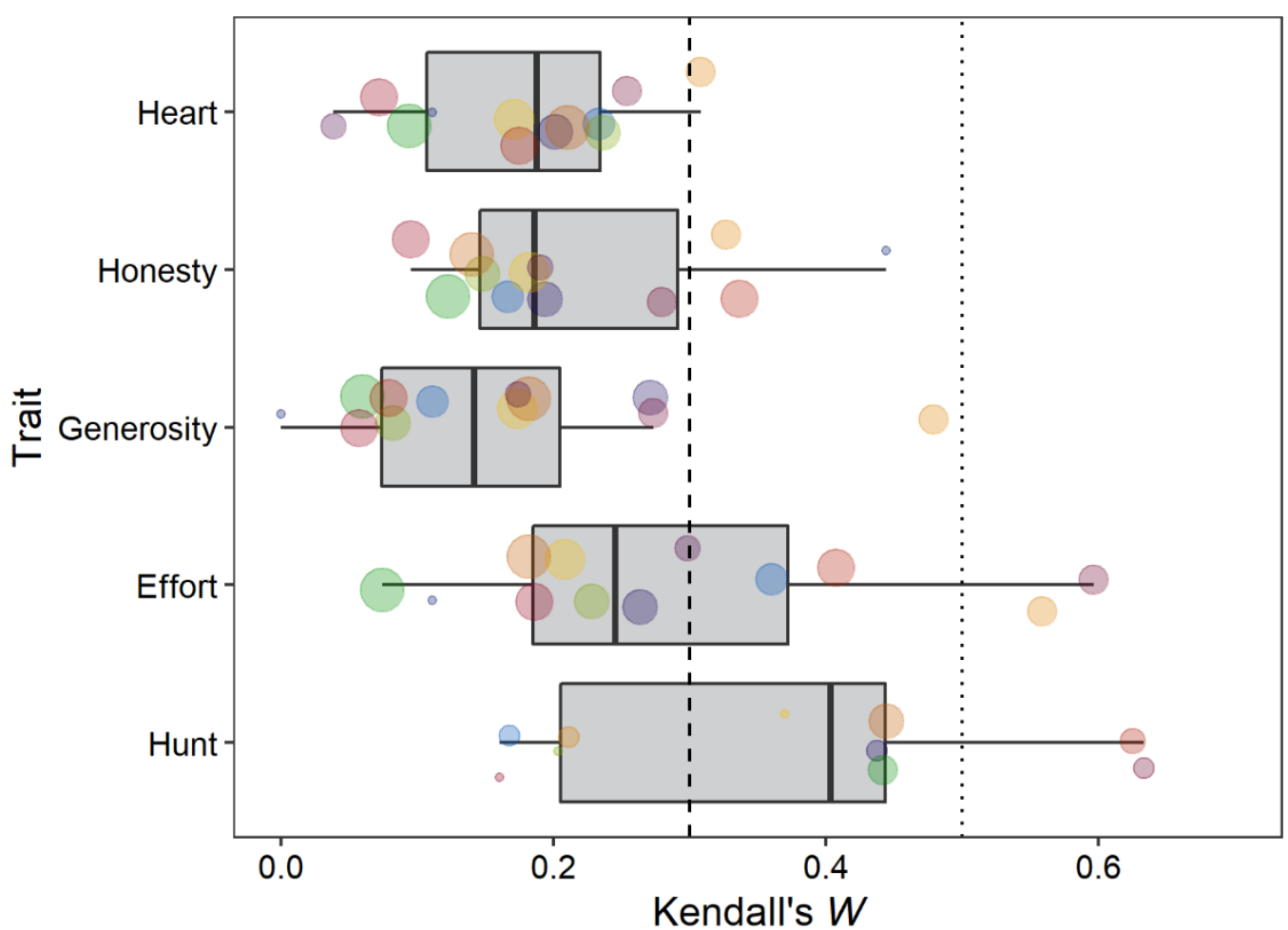

Figure 1. Kendall's $W$ for each trait for each camp with boxplots. The points represent observed values, the size of the points represent the number of subjects ranked, and the color indicates individual camps. The light dashed line is at 0.5 , which is suggested as moderate agreement, and the heavy dashed line is at 0.3 , which is suggested as weak agreement. Two camps had only one man and were not included in the calculation for ranking on hunting ability.

For inferential analyses, we used a transformed value of Kendall's $W$. A transformed Kendall's $W$ has a chi-square distribution such that $m(k-1) W \sim X^{2}(k-1, \lambda)$, where $m$ is the number of judges, $k$ is the number of items being ranked, and $\lambda$ is the non-centrality parameter (Zar, 1996). We fit a linear regression model with a non-central chi-square distribution likelihood estimating $\lambda$ given the transformed $W$ 's for each trait and camp and the number of subjects ranked. This allows us to estimate a population-wide level of agreement within each 
camp; the estimated values were then be used to simulate statistics describing the level of agreement. We included random intercepts for camps, and dummy-coded the traits relative to the good heart rankings. To fit the model, we used 'rjags' (Plummer, 2018) with four chains; the chains had 10,000 iterations burn-in and each chain sampled 10,000 iterations from the posterior distribution.

Table 1 presents the estimated non-centrality parameter for each trait. From these noncentrality parameters, we simulated Kendall's $W$ 's for each trait; because $W$ is dependent on the number of judges and people being ranked, we simulated $W$ for a small $(m=6)$, medium $(m=8)$, and large $(m=10)$ camp. For good heart, generosity, and honesty, even in the small camp, the simulated values fell below the 0.30 value for weak agreement, whereas for effort the values in small camps were near this benchmark and for hunting ability the values in the small camp were above the benchmark for moderate agreement (0.50). In larger camps, the estimated $W$ for every trait falls below 0.30, except for hunting ability, which in medium camps still falls above that benchmark. We also directly compared the estimated non-centrality parameters between each trait (see Table 2) to compare agreement holding camp size constant; Hadza agreed more on rankings of hunting ability and effort than they did on rankings of good heart, generosity, and honesty. 
Table 1. Estimated Non-centrality Parameter and Simulated Kendall's $W$ for each Trait

\begin{tabular}{lrrrr}
\hline Trait & $\lambda$ & $W(m=6)$ & $W(m=8)$ & $W(m=10)$ \\
\hline Heart & $2.19[0.94,3.74]$ & $0.24[0.20,0.29]$ & $0.16[0.14,0.19]$ & $0.12[0.11,0.14]$ \\
Honesty & $1.70[0.63,4.12]$ & $0.24[0.19,0.31]$ & $0.16[0.14,0.20]$ & $0.12[0.11,0.15]$ \\
Generosity & $1.31[0.41,3.04]$ & $0.21[0.18,0.27]$ & $0.15[0.13,0.18]$ & $0.12[0.10,0.13]$ \\
Effort & $3.85[1.62,7.35]$ & $0.30[0.22,0.41]$ & $0.19[0.15,0.25]$ & $0.14[0.12,0.18]$ \\
Hunt & $5.07[2.13,8.83]$ & $0.58[0.36,0.90]$ & $0.33[0.21,0.48]$ & $0.22[0.15,0.31]$
\end{tabular}

Note. Values are modal values from the posterior with $90 \%$ highest density intervals (HDI), intervals, which is the continuous range of the $90 \%$ most plausible values in brackets. $\lambda$ is the estimated non-centrality parameter. $W$ values are simulated from the non-centrality parameter for a camp with $m$ judges. For all traits but hunting, there are $k=m$ subjects; for hunting, $k=m / 2$.

Table 2. Comparisons of Estimated Non-centrality Parameters Between each Trait

\begin{tabular}{lccccr}
\hline & Heart & Honesty & Generosity & Effort & Hunt \\
\hline Heart & - & 0.52 & 0.80 & 0.05 & 0.01 \\
Honesty & 0.49 & - & 0.73 & 0.09 & 0.02 \\
Generosity & 0.20 & 0.27 & - & 0.02 & 0.00 \\
Effort & 0.95 & 0.92 & 0.98 & - & 0.25 \\
Hunt & 1.00 & 0.99 & 1.00 & 0.75 & - \\
Note. Values are proportions of posterior in which $\lambda$ for the trait in the row is greater than the $\lambda$ \\
for the trait in the column. For example, the non-centrality parameter of good heart is greater \\
than the non-centrality parameter for honesty in $52 \%$ of the posterior.
\end{tabular}


We converted the simulated $W$ values to $r$ such that $r=\frac{m W-1}{m-1} ; r$ is the mean Spearman's $\rho$ between each possible pair of judges (Zar, 1996), or the expected agreement between pairs of judges. In a medium sized $(m=8)$ camp, the modal simulated $r$ value for good heart was $r=0.04$ [90\% HDI: 0.02, 0.08], for honesty $r=0.04$ [90\% HDI: 0.02, 0.09], for generosity $r=0.03$ [90\% HDI: 0.01, 0.06], for effort $r=0.08$ [90\% HDI: 0.03, 0.15], and for hunting $r=0.23$ [90\% HDI: $0.10,0.41]$. Figure 2 presents the distribution of simulated $r$ values for each trait for each small, medium, and large camps. These values indicate that judges disagree on their rankings of campmates' character.

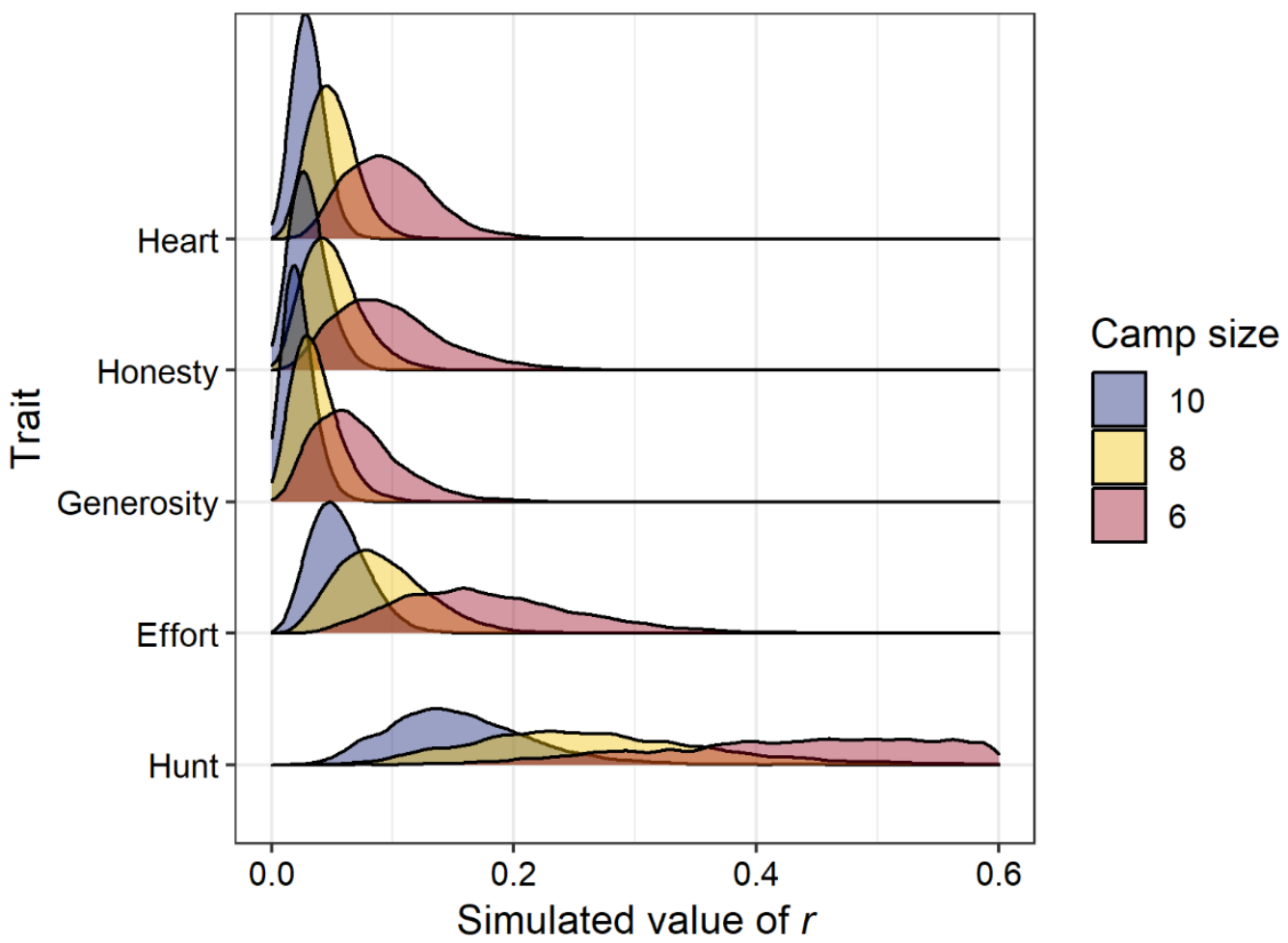

Figure 2. Simulated $r$ values from the estimated non-centrality parameter for each trait. For heart, honesty, generosity, and effort, the number of judges and the number of subjects being ranked are equal to camp size; for hunting, the number of subjects being ranked is half the camp size. 


\section{Do Hadza agree on what makes someone moral?}

To examine which traits Hadza consider important to moral character, we fit an ordered logistic model regressing rankings of good heart on rankings of effort, generosity, and honesty, as well as the subject's sex, age ( $z$-scored), marital status, and the relationship between judge and subject (i.e., self, spouse, kin, or none). Character rankings were centered within each camp such that rank changes are relative to the camp median. We include random intercepts for camp and subject, random slopes for camp and judge for every effect, and random slopes for subject for the effects of character rankings and the relationship between judge and subject. We fit the model using 'brms' (Bürkner, 2017), which implements Stan (Carpenter et al., 2017) to estimate the posterior. We used one chain with 20,000 iterations, the first 10,000 iterations were warm-up, with a step parameter of $\delta=0.95$.

\section{Population-level effects}

Table 3 presents the coefficients for all variables in the model. There was strong evidence that higher rankings on effort and generosity were related to higher rankings on good heart, while there was suggestive evidence that higher rankings on honesty were related to higher rankings on good heart. There was some evidence that older Hadza had higher rankings on good heart, otherwise demographic variables did not relate to rankings on good heart. We simulated rankings of good heart as a function of rankings on effort, generosity, and honesty in the largest camp $(n=$ 12) and computed the expected difference in good heart ranking between the highest and lowest ranked person on each trait. The modal difference for effort was 3.2 (90\% HDI: 1.9, 4.4) ranks, for generosity 1.7 (90\% HDI: 0.2, 3.0) ranks, and for honesty 0.9 (90\% HDI: $-0.3,2.6)$ ranks. Figure 3 presents the full range of simulated rankings. 
Table 3. Model Regressing Good Heart Ranking on Character Rankings and Subject

Demographics

\begin{tabular}{lccc}
\hline Coefficient & $b$ & $90 \%$ HDI & $\%>0$ \\
\hline Effort & 0.24 & $0.13-0.35$ & 97.2 \\
Generosity & 0.13 & $0.02-0.24$ & 88.1 \\
Honesty & 0.08 & $-0.04-0.20$ & 54.3 \\
Female & 0.21 & $-1.37-1.62$ & 76.5 \\
Married & 0.29 & $-0.31-0.73$ & 85.4 \\
Age & 0.22 & $-0.13-0.54$ & 79.1 \\
Spouse & 0.32 & $-0.38-1.22$ & 78.4 \\
Kin & 0.34 & $-0.39-1.02$ & 56.1 \\
Self & 0.00 & $-0.87-1.06$ &
\end{tabular}

Note. The coefficient estimate is the mode of the posterior distribution. The $90 \%$ highest

posterior density interval (HDI) is the narrowest interval containing $90 \%$ of the posterior, or the $90 \%$ most plausible coefficient estimates. The last column is the percent of the posterior greater than zero. 

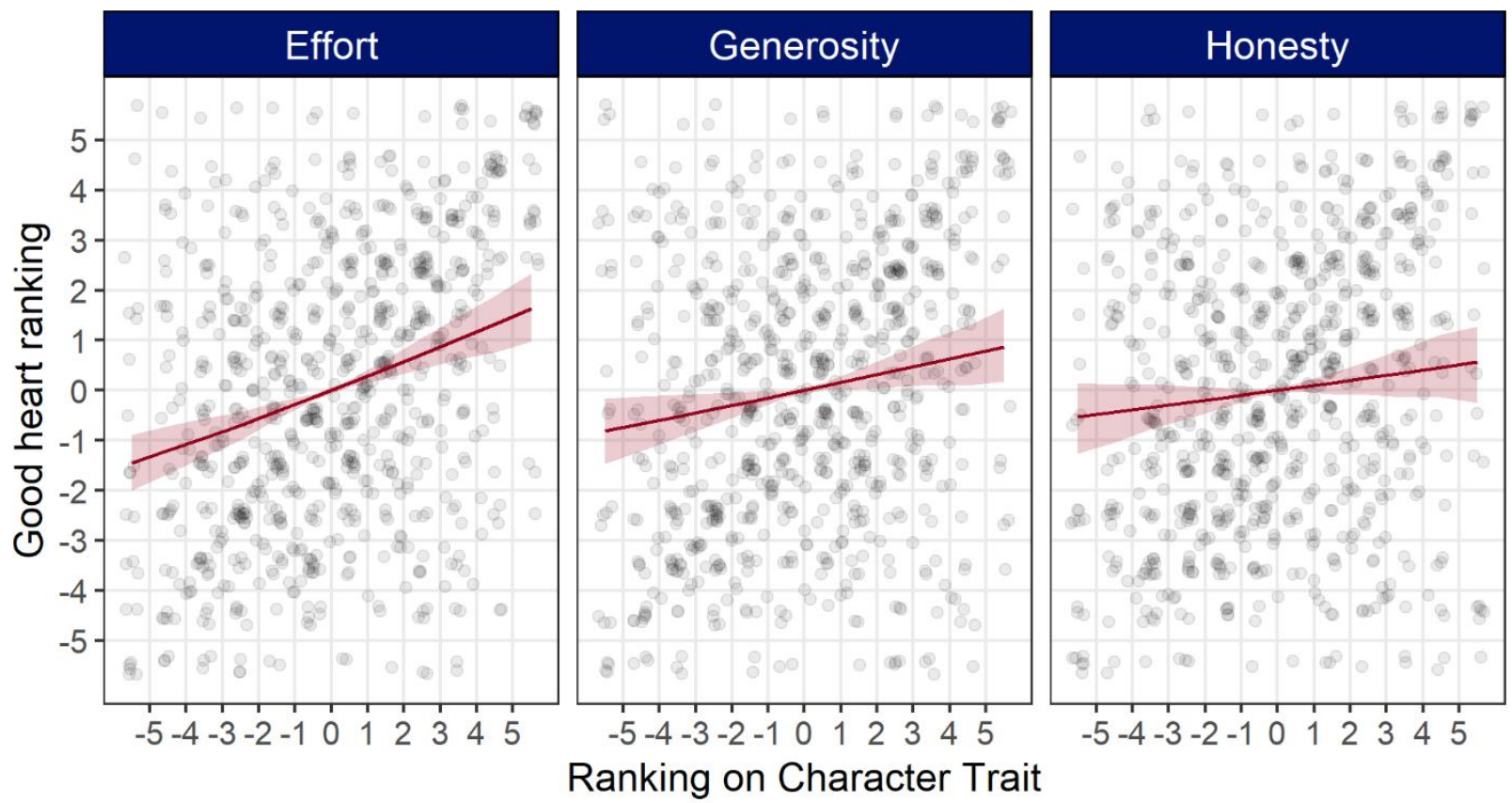

Figure 3. Ranking on good heart by ranking on each character trait, centered within camp. Predictions were simulated by generating 10,000 values from an ordered logistic distribution for each ranking and predicted ranking was the mean of these generated values. The line is the modal simulated value and the shaded region is the $90 \%$ credible interval.

\section{Judge-level effects}

To determine whether judges agreed on how much the specific traits contributed to an individual's global character, we examined variation between judges on the relationship between character rankings and good heart rankings. First, we fit a series of eight models including varying slopes for judges for none, one, two, or all of the character traits; the eight models were otherwise identical. This allowed us to examine whether including extra parameters to estimate varying slopes for judges was worth the improved fit. Table 4 presents fit statistics for these models, including the Akaike weight. The Akaike weight is the probability that a model would best predict a new sample of data compared to all the other models under consideration 
(McElreath, 2016). The Akaike weight is distributed across most of the models. Models including random slopes for effort had 0.35 of the weight, for generosity had 0.54 of the weight, and for honesty had 0.95 of the weight; this indicates that the model most likely to best estimate the data is likely to include disagreement between judges on the contribution of honesty to good heart, and is less likely to include disagreement on the contribution of effort and generosity to good heart. Rather than selecting one model as the best fitting, we constructed a weightedaverage posterior using the Akaike weights (McElreath, 2016) and examined the variation between judges using that posterior.

Table 4. Fit of Models Regressing Good Hearts Rankings on Character Rankings

\begin{tabular}{|c|c|c|c|c|}
\hline Model & WAIC & $S E_{\mathrm{WAIC}}$ & $p_{\text {WAIC }}$ & Akaike weight \\
\hline Baseline & 1760.77 & 29.80 & 100.90 & 0.02 \\
\hline Effort & 1761.11 & 29.61 & 107.82 & 0.01 \\
\hline Generosity & 1761.14 & 29.58 & 113.17 & 0.01 \\
\hline Honesty & 1754.80 & 31.13 & 123.21 & 0.30 \\
\hline Effort + Generosity & 1761.29 & 29.50 & 118.45 & 0.01 \\
\hline Effort + Honesty & 1756.49 & 30.99 & 128.09 & 0.13 \\
\hline Generosity + Honesty & 1754.68 & 30.99 & 139.14 & 0.32 \\
\hline Full & 1755.60 & 31.03 & 142.67 & 0.20 \\
\hline
\end{tabular}

Note. Model names refer to what character traits in the model had random slopes for judges.

WAIC is the widely-applicable information criterion estimate; a lower number indicates a better fitting model. $S E_{\text {WAIC }}$ is the standard error of the WAIC estimate, $p_{\text {WAIC }}$ is the effective number of parameters in the model, and the Akaike weight is computed from WAIC and is the probability that the model will best predict a new set of data among the considered models. 
In the weighted-average posterior, the modal $\sigma_{\text {Judge }}$ of varying slopes for effort was 0.00 (90\% HDI: 0, 0.13), for generosity 0.00 (90\% HDI: 0.00, 0.26), and for honesty 0.24 (90\% HDI: $0.09,0.38)$. We also compared the $\sigma_{\text {Judge }}$ to the population coefficient; the percent of the posterior for which the $\sigma_{J u d g e}$ was greater than the population coefficient for effort was $2.0 \%$, for generosity $40.2 \%$, and for honesty $88.8 \%$; there was good evidence that knowing a particular judge's belief of the role of honesty in character provided more information than knowing the population's belief, but this was not the case for effort and generosity. Finally, as another way to examine consensus, we computed the expected proportion of judges to have a negative slope between rankings on each trait and rankings on good heart. The modal expected proportion of negative slopes for effort was 0.00 (90\% HDI: 0.00, 0.04), for generosity 0.00 (90\% HDI: 0.00, 0.38), and for honesty 0.38 (90\% HDI: 0.00, 0.51). Again, for honesty, even though at the population level more honest Hadza were ranked higher on good heart, a number of judges ranked more honest Hadza lower on good heart. Figure 4 presents at the mean of the posterior the simulated variation between judges across rankings. 


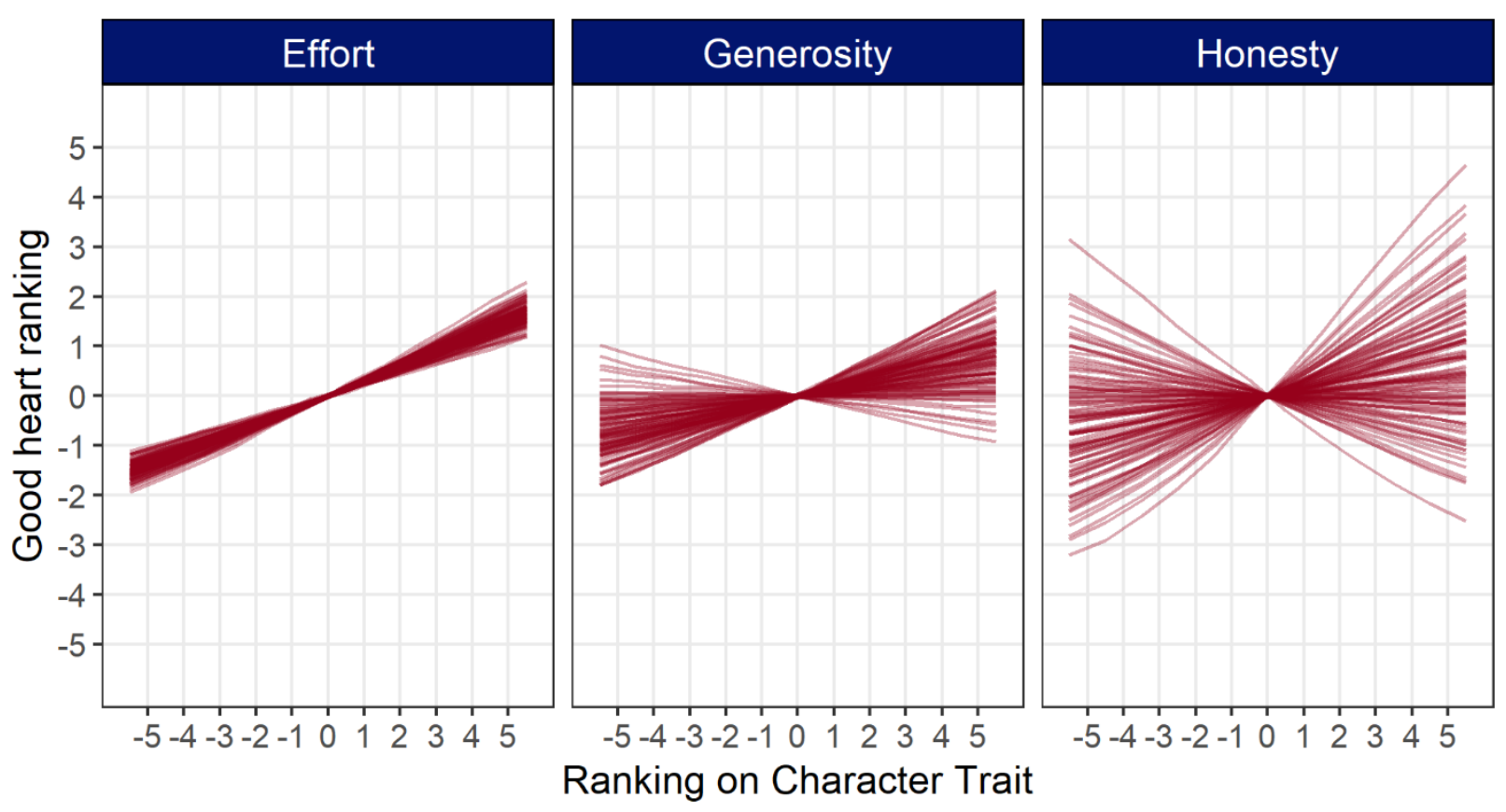

Figure 4. Ranking on good heart by ranking on each character trait, centered within camp. Predictions were simulated by generating 10,000 values from an ordered logistic distribution for each ranking and the mean of these generated values were predicted ranking. Each line is a simulated judge's slope taken from the mean of weighted-average posterior.

\section{Discussion}

In WEIRD societies, people evaluate the moral character of others and use those perceptions to decide with whom to interact. Underscoring the importance of character in these populations, independent observers agree on how moral others are (Helzer et al., 2014). But is this universal? To answer this, we asked if Hadza hunter-gatherers agree on who is moral and what traits make someone moral. The Hadza disagree on which of their campmates have a good heart, are generous, and are honest, and agree more on which campmates are hard working (effort) and produce the most food (hunting ability). At the level of the population, hard work, generosity, and honesty contribute to global character; however, there is variation between 
Hadza judges on how much honesty contributes to global character, though judges agree more on how much hard work and generosity contribute to character. Overall, these results suggest that Hadza use some of the same criteria — hard work and generosity—for evaluating moral character, but disagree on who displays those traits, leading to disagreement on global character perceptions.

Agreement between independent observers on ratings about a trait is taken as evidence for that trait existing because raters are likely observing the same behaviors despite being in different situations (Kenrick \& Funder, 1988). The disagreement between Hadza judges on character traits suggests that Hadza do not have stable variation in moral dispositions. ${ }^{2}$ However, disagreement does not definitively rule out the existence of moral character. For example, the Hadza may have been unwilling to make assessments about their campmates' character, though notably we do see agreement on hunting ability, which is highly valued in the Hadza. Or there could be disagreement because there are not many opportunities to display moral behavior; however, it should be easy to observe moral behavior because they live together in small groups and depend on each other for survival. Finally, another alternative is that Hadza display consistent moral behavior to specific people; that is, a person could always be generous to one campmate and selfish to another campmate, leading to disagreement between campmates in evaluations of moral character. Future research exploring the stability of judge-subject rankings across time could address this alternative interpretation.

One alternative interpretation of the data is that the Hadza can agree on moral character, and in fact they do have moral dispositions, but that our measure is unreliable and cannot detect agreement. A good measure measuring a phenomenon that does not exist and a bad measure

\footnotetext{
2 To be clear, the claim is not that the Hadza are immoral, but that an individuals' moral behavior varies across time, changing to adapt to local circumstances.
} 
measuring a phenomenon that does exist will produce the same result: noise. However, we argue there are two reasons to suspect that our measure would be reliable enough to detect agreement on moral character if it existed. First, we were able to detect moderate relationships between the specific character traits and moral character, indicating reliability was not so low as to be unable to detect any effects. Second, we did find moderate agreement on hard work and hunting ability. And in fact, given what we know about the noisy relationship between hunting returns and hunting reputation (Stibbard-Hawkes et al., 2018), the fact that we were able to detect agreement suggests low reliability can not fully explain the disagreement in perceptions of moral character.

It may seem that hunting ability would be easily observable, but in the anthropological literature, this is notoriously difficult to measure, and because of this hunting reputation is criticized as a measure of hunting success (Hill \& Kintigh, 2009). First, hunting ability is rarely directly observed, as most hunting happens alone. And second, there is high variance in hunting returns, in which men return to camp with nothing on most days, but occasionally (about $3 \%$ of days) bring in large game (Hawkes, O’Connell, \& Blurton-Jones, 1991). In fact, for anthropologists to reliably estimate hunting ability using hunting returns, they need 200 to 600 days of observations (Hill \& Kintigh, 2009). Despite this, in our study and others (StibbardHawkes et al., 2018), the Hadza are able to agree on who the best hunters are, and hunting reputation does relate to proxies of actual hunting ability, such as strength, accuracy, and ecological knowledge (Apicella, 2014; Stibbard-Hawkes et al., 2018). This suggests that if there are moral dispositions among the Hadza, the signal is much weaker than that of hunting ability, which is itself a noisy signal (Stibbard-Hawkes, 2019). And in fact, if it is this hard to detect moral dispositions, it then raises the question of whether the Hadza can reliably determine character enough to provide useful social information. 
Data measuring morally-relevant behavior, such as generosity, further suggest a lack of stable variation in moral dispositions in the Hadza and other non-WEIRD populations. In a longitudinal study, a Hadza's previous generosity in an economic game did not predict their subsequent contributions, and instead the only significant predictor was how much his or her campmates contribute (Smith et al., 2018). And in a small study $(n=12)$ of the Tsimane of Bolivia, generosity in a dictator game in one year did not predict generosity in a later year (Gurven, 2014).

These results further support recent research finding that character and moral reputation do not play a role in Hadza campmate preferences. When asked who they prefer to live with, Hadza do not choose the most generous people, whether generosity is measured using an economic game (Apicella, Marlowe, Fowler, \& Christakis, 2012) or via reputation (Smith \& Apicella, 2019). Rather, Hadza prefer to live with better hunters (Smith \& Apicella, 2019; Wood, 2006). If moral behavior changes across time and situations as our results here suggest, then choosing campmates based on their current behavior is useless. Instead, traits related to productivity, such as being a hard worker or a good hunter, may become more important in campmate preferences (Barclay, 2016); if everyone is expected to share because of strong norms, such as in the Hadza, then choosing productive campmates is more important. And in fact, a preference for productive partners may influence friendships in Western societies. US undergraduates and online workers prefer partners in economic games and are more generous to partners who are perceived to be more productive, even though it is irrelevant to the game (Eisenbruch, Grillot, Maestripieri, \& Roney, 2016; Eisenbruch \& Roney, 2017). The effect of a productivity preference in various relationships may be a fruitful area for future research. 
Throughout the latter half of the $20^{\text {th }}$ century, the situationist paradigm in social psychology casted doubt on the existence of moral character. However, more recent research in moral psychology has argued that moral character does in fact exist (Fleeson, Furr, Jayawickreme, Meindl, \& Helzer, 2014). In Western societies, people agree on who is moral (Helzer et al., 2014), and perceptions of moral character play an important role in social cognition (Goodwin, 2015; Landy \& Uhlmann, 2018). Our results here question the universality of moral character and its centrality in social life, and highlights the importance of cross-cultural research using underrepresented samples. By conducting research with populations in a variety of socio-ecologies, we can better understand the variation in our moral psychology. 


\section{References}

Apicella, C. L. (2014). Upper-body strength predicts hunting reputation and reproductive success in Hadza hunter-gatherers. Evolution and Human Behavior, 35(6), 508-518. https://doi.org/10.1016/j.evolhumbehav.2014.07.001

Apicella, C. L. (2018). High levels of rule-bending in a minimally religious and largely egalitarian forager population. Religion, Brain \& Behavior, 8(2), 133-148. https://doi.org/10.1080/2153599X.2016.1267034

Apicella, C. L., Marlowe, F. W., Fowler, J. H., \& Christakis, N. A. (2012). Social networks and cooperation in hunter-gatherers. Nature, 481(7382), 497-501. https://doi.org/10.1038/nature10736

Barclay, P. (2016). Reputation. In The Handbook of Evolutionary Psychology (pp. 810-828). Hoboken: Wiley.

Barrett, H. C., Bolyanatz, A., Crittenden, A. N., Fessler, D. M. T., Fitzpatrick, S., Gurven, M., ... Laurence, S. (2016). Small-scale societies exhibit fundamental variation in the role of intentions in moral judgments. Proceedings of the National Academy, 113(17), 4688-4693.

Blurton-Jones, N. (2016). Deomgraphy and Evolutionary Ecology of Hadza Hunter-Gatherers. Cambridge: Cambridge University Press.

Brambilla, M., Rusconi, P., Sacchi, S., \& Cherubini, P. (2011). Looking for honesty: The primary role of morality (vs. sociability and competence) in information gathering. European Journal of Social Psychology, 41(2), 135-143. https://doi.org/10.1002/ejsp.744

Bürkner, P.-C. (2017). brms : An R Package for Bayesian Multilevel Models Using Stan. Journal of Statistical Software, 80(1), 1-28. https://doi.org/10.18637/jss.v080.i01

Carpenter, B., Gelman, A., Hoffman, M. D., Lee, D., Goodrich, B., Betancourt, M., ... Riddell, 
A. (2017). Stan : A Probabilistic Programming Language. Journal of Statistical Software, 76(1). https://doi.org/10.18637/jss.v076.i01

Cohen, T. R., Panter, A. T., Turan, N., Morse, L., \& Kim, Y. (2013). Agreement and similarity in self-other perceptions of moral character. Journal of Research in Personality, 47(6), 816830. https://doi.org/10.1016/j.jrp.2013.08.009

Cottrell, C. A., Neuberg, S. L., \& Li, N. P. (2007). What do people desire in others? A sociofunctional perspective on the importance of different valued characteristics. Journal of Personality and Social Psychology, 92(2), 208-231. https://doi.org/10.1037/00223514.92.2.208

Curry, O. S., Mullins, D. A., \& Whitehouse, H. (2019). Is it good to cooperate? Testing the theory of morality-as-cooperation in 60 societies. Current Anthropology, 60(1), 47-69. https://doi.org/10.1086/701478

Darley, J. M., \& Batson, C. D. (1973). "From Jerusalem to Jericho": A study of situational and dispositional variables in helping behavior. Journal of Personality and Social Psychology, 27(1), 100-108. https://doi.org/10.1037/h0034449

Darley, J. M., \& Latané, B. (1968). Bystander intervention in emergencies: Diffusion of responsibility. Journal of Personality and Social Psychology, 8(4, Pt.1), 377-383. https://doi.org/10.1037/h0025589

Doris, J. M. (2002). Lack of Character: Personality and Moral Behavior. New York: Cambridge University Press.

Eisenbruch, A. B., Grillot, R. L., Maestripieri, D., \& Roney, J. R. (2016). Evidence of partner choice heuristics in a one-shot bargaining game. Evolution and Human Behavior, 37(6), 429-439. https://doi.org/10.1016/j.evolhumbehav.2016.04.002 
Eisenbruch, A. B., \& Roney, J. R. (2017). The Skillful and the stingy: Partner choice decisions and fairness intuitions suggest human adaptation for a biological market of cooperators. Evolutionary Psychological Science, 3(4), 364-378. https://doi.org/10.1007/s40806-0170107-7

Ellemers, N., van der Toorn, J., Paunov, Y., \& van Leeuwen, T. (2019). The psychology of morality: A review and analysis of empirical studies published from 1940 through 2017. Personality and Social Psychology Review, 108886831881175. https://doi.org/10.1177/1088868318811759

Everett, J. A. C., Faber, N. S., Savulescu, J., \& Crockett, M. J. (2018). The costs of being consequentialist: Social inference from instrumental harm and impartial beneficence. Journal of Experimental Social Psychology, 79, 200-216. https://doi.org/10.1016/j.jesp.2018.07.004

Fleeson, W., Furr, R. M., Jayawickreme, E., Meindl, P., \& Helzer, E. G. (2014). Character: The prospects for a personality-based perspective on morality. Social and Personality Psychology Compass, 8(4), 178-191. https://doi.org/10.1111/spc3.12094

Frimer, J. A., Biesanz, J. C., Walker, L. J., \& MacKinlay, C. W. (2013). Liberals and conservatives rely on common moral foundations when making moral judgments about influential people. Journal of Personality and Social Psychology, 104(6), 1040-1059. https://doi.org/10.1037/a0032277

Gamer, M., Lemon, J., \& Fellows, I. (2012). irr: Various coefficients of interrater reliability and agreement. Retrieved from https://cran.r-project.org/package=irr

Goodwin, G. P. (2015). Moral character in person perception. Current Directions in Psychological Science, 24(1), 38-44. 
Goodwin, G. P., Piazza, J., \& Rozin, P. (2014). Moral character predominates in person perception and evaluation. Journal of Personality and Social Psychology, 106(1), 148-168. https://doi.org/10.1037/a0034726

Gurven, M. (2014). The Tsimane rarely punish: An experimental investigation of dictators, ultimatums, and punishment. In J. Ensminger \& J. Henrich (Eds.), Experimenting with Social Norms: Fairness and Punishment in Cross-Cultural Perspective (pp. 197-224). New York: Russell Sage Foundation.

Gurven, M., von Rueden, C., Massenkoff, M., Kaplan, H., \& Vie, M. L. (2013). How universal is the Big Five? Testing the five-factor model of personality variation among forager-farmers in the Bolivian Amazon. Journal of Personality and Social Psychology, 104(2), 354-370. https://doi.org/10.1037/a0030841

Haidt, J., Koller, S. H., \& Dias, M. G. (1993). Affect, culture, and morality, or is it wrong to eat your dog? Journal of Personality and Social Psychology, 65(4), 613-628. https://doi.org/10.1037/0022-3514.65.4.613

Harman, G. (2003). No character or personality. Business Ethics Quarterly, 13(1), 87-94. https://doi.org/10.5840/beq20031316

Hawkes, K., O’Connell, J. F., \& Blurton-Jones, N. (1991). Hunting income patterns among the Hadza: big game, common goods, foraging goals and the evolution of the human diet. Philosophical Transactions of the Royal Society of London. Series B: Biological Sciences, 334(1270), 243-251. https://doi.org/10.1098/rstb.1991.0113

Helzer, E. G., Furr, R. M., Hawkins, A., Barranti, M., Blackie, L. E. R., \& Fleeson, W. (2014). Agreement on the perception of moral character. Personality and Social Psychology Bulletin, 40(12), 1698-1710. https://doi.org/10.1177/0146167214554957 
Henrich, J. (2006). Costly punishment across human societies. Science, 312(5781), 1767-1770. https://doi.org/10.1126/science.1127333

Henrich, J., Heine, S., \& Norenzayan, A. (2010). The WEIRDest people in the world. Behavioral and Brain Sciences, 33(2), 1-75.

Hill, K. R., \& Kintigh, K. (2009). Can anthropologists distinguish good and poor hunters? Implications for hunting hypotheses, sharing conventions, and cultural transmission. Current Anthropology, 50(3), 369-378. https://doi.org/10.1086/597981

Hill, K. R., Walker, R. S., Božičević, M., Eder, J., Headland, T., Hewlett, B., ... Wood, B. (2011). Co-Residence Patterns in Hunter-Hatherer Societies Show Unique Human Social Structure. Science, 331, 1286-1289. https://doi.org/10.1126/science.1199071

Hill, K. R., Wood, B. M., Baggio, J., Hurtado, A. M., \& Boyd, R. T. (2014). Hunter-Gatherer Inter-band interaction rates: Implications for cumulative culture. PLoS ONE, 9(7), e102806. https://doi.org/10.1371/journal.pone.0102806

Kay, M. (2018). tidybayes: Tidy data and geoms for Bayesian models.

Kenrick, D. T., \& Funder, D. C. (1988). Profiting from controversy: Lessons from the personsituation debate. American Psychologist, 43(1), 23-34. https://doi.org/10.1037/0003066X.43.1.23

Kruschke, J. K., \& Liddell, T. M. (2018a). Bayesian data analysis for newcomers. Psychonomic Bulletin \& Review, 25(1), 155-177. https://doi.org/10.3758/s13423-017-1272-1

Kruschke, J. K., \& Liddell, T. M. (2018b). The Bayesian New Statistics: Hypothesis testing, estimation, meta-analysis, and power analysis from a Bayesian perspective. Psychonomic Bulletin \& Review, 25(1), 178-206. https://doi.org/10.3758/s13423-016-1221-4

Landy, J. F., Piazza, J., \& Goodwin, G. P. (2016). When it's bad to be friendly and smart: The 
desirability of sociability and competence depends on morality. Personality and Social Psychology Bulletin, 42(9), 1272-1290. https://doi.org/10.1177/0146167216655984

Landy, J. F., \& Uhlmann, E. (2018). Morality is personal. In K. Gray \& J. Graham (Eds.), Atlas of Moral Psychology (p. 121). New York: Guilford Press.

Latané, B., \& Darley, J. M. (1968). Group inhibition of bystander intervention in emergencies. Journal of Personality and Social Psychology, 10(3), 215-221. https://doi.org/10.1037/h0026570

Lukaszewski, A., Gurven, M., von Rueden, C., \& Schmitt, D. P. (2017). What explains personality covariation? A test of the socioecological complexity hypothesis. Social Psychological and Personality Science, 8(8), 943-952.

https://doi.org/10.1177/1948550617697175

Marlowe, F. W. (2010). The Hadza: Hunter-Gatherers of Tanzania. Berkley: University of California Press.

Martin, J. W., \& Cushman, F. (2015). To punish or to leave: Distinct cognitive processes underlie partner control and partner choice behaviors. PLOS ONE, 10(4), 9-14. https://doi.org/10.1371/journal.pone.0125193

McElreath, R. (2016). Statistical Rethinking. Boca Raton, FL: Taylor \& Francis.

McNamara, R. A., Willard, A. K., Norenzayan, A., \& Henrich, J. (2019). Weighing outcome vs. intent across societies: How cultural models of mind shape moral reasoning. Cognition, 182, 95-108. https://doi.org/10.1016/j.cognition.2018.09.008

Plummer, M. (2018). rjags: Bayesian graphical models using MCMC. Retrieved from https://cran.r-project.org/package=rjags

Purzycki, B. G., Pisor, A. C., Apicella, C. L., Atkinson, Q., Cohen, E., Henrich, J., ... Xygalatas, 
D. (2018). The cognitive and cultural foundations of moral behavior. Evolution and Human Behavior, 39(5), 490-501. https://doi.org/10.1016/j.evolhumbehav.2018.04.004

R, (CoreTeam). (2017). R: A language and environment for statistical computing.

Smith, K. M., \& Apicella, C. L. (2019). Partner choice in human evolution: The role of character, hunting ability, and reciprocity in Hadza campmate selection. https://doi.org/10.31234/osf.io/35tch

Smith, K. M., Larroucau, T., Mabulla, I. A., \& Apicella, C. L. (2018). Hunter-Gatherers maintain assortativity in cooperation despite high levels of residential change and mixing. Current Biology, 28(19), 3152-3157.e4. https://doi.org/10.1016/j.cub.2018.07.064

Smith, K. M., Olkhov, Y. M., Puts, D. A., \& Apicella, C. L. (2017). Hadza men with lower voice pitch have a better hunting reputation. Evolutionary Psychology, 15(4). https://doi.org/10.1177/1474704917740466

Stibbard-Hawkes, D. N. E. (2019). Costly signaling and the handicap principle in hunter-gatherer research: A critical review. Evolutionary Anthropology: Issues, News, and Reviews. https://doi.org/10.1002/evan.21767

Stibbard-Hawkes, D. N. E., Attenborough, R. D., \& Marlowe, F. W. (2018). A noisy signal: To what extent are Hadza hunting reputations predictive of actual hunting skills? Evolution and Human Behavior, 39(6), 639-651. https://doi.org/10.1016/j.evolhumbehav.2018.06.005

Strohminger, N., Knobe, J., \& Newman, G. (2017). The True Self: A psychological concept distinct from the self. Perspectives on Psychological Science, 12(4), 551-560. https://doi.org/10.1177/1745691616689495

Strohminger, N., \& Nichols, S. (2014). The essential moral self. Cognition, 131(1), 159-171. https://doi.org/10.1016/j.cognition.2013.12.005 
Thalmayer, A. G., Saucier, G., Srivastava, S., Flournoy, J. C., \& Costello, C. K. (2019). Ethicsrelevant values in adulthood: Longitudinal findings from the life and time study. Journal of Personality. https://doi.org/10.1111/jopy.12462

van der Lee, R., Ellemers, N., Scheepers, D., \& Rutjens, B. T. (2017). In or out? How the perceived morality (vs. competence) of prospective group members affects acceptance and rejection. European Journal of Social Psychology, 47(6), 748-762. https://doi.org/10.1002/ejsp.2269

Wickham, H. (2017). tidyverse: Easily install and load the "tidyverse.” Retrieved from https://cran.r-project.org/package=tidyverse

Wilke, C. O. (2018). ggridges: Ridgeline plots in "ggplot2.” Retrieved from https://cran.rproject.org/package=ggridges

Wood, B. M. (2006). Prestige or provisioning? A test of foraging goals among the Hadza. Current Anthropology, 47(2), 383-387. https://doi.org/10.1086/503068

Zar, J. H. (1996). Biostatistical Analysis (3rd ed.). Upper Saddle River: Prentice-Hall. 